EESTI NSV TEADUSTE AKADEEMIA TOIMETISED. XI KOIDE

FOOSIKALIS-MATEMAATILISTE JA TEHNILISTE TEADUSTE SEERIA. 1962, NR. 3

ИЗВЕСТИЯ АКАДЕМИИ НАУК ЭСТОНСКОЙ ССР. ТОМ ХІ

СЕРИЯ ФИЗИКО-МАТЕМАТИЧЕСКИХ И ТЕХНИЧЕСКИХ НАУК. 1962, No 3

\title{
ТЕОРИЯ ПРОСВЕТЛЕННОГО СВЕТОФИЛЬТРА ТИПА ФАБРИ-ПЕРО
}

\author{
П. КАРД, \\ член-корреспондент Академии наук Эстонской ССР
}

Введение

Обычный, непросветленный светофильтр типа Фабри-Перо состоит, как известно, из двух полупрозрачных металлических слоев, между которыми находится тонкий диэлектрический слой. Все три слоя заключены между двумя стеклянными пластинками. Обычно обе пластинки делаются одинаковыми, равно как и металлические слои. Тогда фильтр является симметричным, что мы ниже и будем предполагать. Будем также считать падение света нормальным, хотя наша теория фактически применима и в случае слегка наклонного падения света. Поэтому ею можно пользоваться и для эталона Фабри-Перо, который отличается от фильтра только гораздо большей толщиной промежуточного слоя.

Оптические свойства симметричного светофильтра типа Фабри-Перо определяются формулой

$$
\frac{1}{D}=\left(1+\frac{A_{1}}{D_{1}}\right)^{2}+\frac{4 R_{1}}{D_{1}^{2}} \sin ^{2}\left(\alpha-\mu_{1}+v_{1}\right)
$$

где $D$ - энергетический коэффициент пропускания фильтра,

$$
\alpha=k n h \text {, }
$$

$k=\frac{2 \pi}{\lambda}$ - волновое число в вакууме, а $n$ и $h$ - показатель преломления и толщина промежуточного слоя. Индекс 1 относится к полуфильтру, т. е. к одному металлическому слою, и притом при падении на него света из промежуточного слоя. $R_{1}, D_{1}, A_{1}$ суть энергетические коэффициенты отражения, пропускания и поглощения полуфильтра, а $\mu_{1}$ и $v_{1}$ суть фазы величин $a_{1} \equiv \frac{r_{1}}{d_{1}}$ и $b_{1} \equiv \frac{1}{d_{1}}$, где $r_{1}$ и $d_{1}-$ амплитудные коэффициенты отражения и пропускания полуфильтра, т. е.

$$
\left.\begin{array}{l}
a_{1}=\sqrt{\frac{R_{1}}{D_{1}} e^{i \mu_{1}},} \\
b_{1}=\frac{1}{\sqrt{D_{1}}} e^{i v_{1}} \cdot
\end{array}\right\}
$$

Подчеркнем еще раз, что $R_{1}, A_{1}, a_{1}$ и $\mu_{1}$ относятся к падению света из промежуточного слоя на металлический слой, а не в обратном направлении, из стеклянной пластинки. Иными словами, эти величины относятся ко второму (считая в направлении падения света) металлическому слою. Что же касается $D_{1}, b_{1}$ и $v_{1}$, то эти величины от направления падения света, как известно, не зависят. Отметим, наконец, что в фор- 
муле (1) отражение света на наружных поверхностях стеклянных пластинок не учитывается, поскольку этот фактор в вопросе о просветлении фнльтра несущественен.

Из формулы (1) вытекает, что в середине полосы пропускания фильтра должно удовлетворяться условие

$$
\alpha-\mu_{1}+v_{1}=p \pi, \quad p-\text { целое, }
$$

определяющее необходимую толщину промежуточного слоя.

Тогда

$$
D_{\max }=\frac{1}{\left(1+\frac{A_{1}}{D_{1}}\right)^{2}}
$$

Это значит, что чем меньше отношение $\frac{A_{1}}{D_{1}}$, тем больше $D_{\max }$. Кроме того, из форму лы (1) следует, что чем больше величина $\frac{R_{1}}{D_{1}^{2}}$, тем у́же полоса пропускания. Обоим требованиям можно удовлетворить, очевидно, лишь компромиссным образом. Чтобы унеличить $D_{\max }$, нужно уменьшить $A_{1} / D_{1}$, но так как коэффициент поглощения тонкого металлического слоя слабо зависит от его толщины $*$ уменьшение $A_{1} / D_{1}$ возможно только путем увеличения $D_{1}$ (т. е. уменьшения толщины металлического слоя); но тогда $R_{1} / D_{1}^{2}$ резко уменьшается и ширина полосы пропускания возрастает.

Отсюда вытекает, что уменьшение отношения $A_{1} / D_{1}$ при неизменной толщине металлического слоя является весьма актуальной задачей. Подобная задача, которую мы называем задачей просветления поглощающих интерференционных пленок, может быть сформулирована не только для фнльтра, но и для любой поглощающей пленки. В самом общем виде она рассматривалась автором ранее в нескольких статьях $\left[{ }^{1-3}\right]$ для произвольной заданной или синтезируемой пленки. В настоящей статье мы применим полученные в этих статьях общие результаты к фильтру типа Фабри-Перо. Нашей целью является приведение данных общей теории к виду, удобному для непосредственной практической реализации.

\section{1. Внутреннее и внешнее просветление}

В случае пленки, содержащей более одного поглощающего слоя, нужно, вообще го. воря, различать внутреннее и внешнее просветление. Дело в том, что для получения абсолютного минимума отношения $A / D$ пленки, имеющей заданные поглощающие слои, нужно удовлетворить нескольким условиям (см. работу [3]), относящимся отчасти к выбору прослойки между поглощающими слоями, отчасти же к выбору дополнительной прослойки между последним поглощающим слоем и подложкой. Первые условия мы называем условиями внутреннего, вторые - внешнего просветления.

Так как светофильтр типа Фабри-Перо содержит два металлических слоя, то єго просветление тоже может быть как внешним, так и внутренним. С переого взгляда кажется, однако, неясным, необходимо ли просветление всего фильтра как целого для улучшения его качества. По формуле (1) мы видели, что решающее значение для качества фильтра имеет лишь отношение $A_{1} / D_{1}$ для полуфйльра (в середине полосы пропускания). Это значит, что полуфильтр должен быть просветлен. Для всего же фнльтра как целого формула (1) требования просветленности непосредственно не содержит. Однако, как мы увидим в следующем разделе, просветление полуфильтра равносильно при определенных условиях просветлению всего фильтра. Следовательно, требование просветленности полуфильтра влечет за собой автоматически просветленность всего фильтра.

- Сильная зависимость $A_{1}$ от толщины наблюдается только у очень тонких слоев, которые в светофильтрах обычно не применяются. 


\section{2. Просветление фильтра типа Фабри-Перо}

Итак, покажем, что условие минимума $A_{1} / D_{1}$, т. е. условие, чтобы (второй) полуфильтр был просветлен, влечет за собой автоматически выполнение всех условий внешнего и внутреннего просветления фильтра, при том лишь дополнительном условии, чтобы фильтр оставался симметричным. Поэтому, введя между вторым металлическим слоем и второй стеклянной пластинкой (подложкой) прослойку, просветляющую этот слой, мы должны ввести такую же прослойку симметрично и между первым металлическим слоем и первой стеклянной пластинкой. Тогда формула (1) сохранит свой вид, с той только разницей, что полуфильтр более не является отдельным металлическим слоем, а представляет собой комбинацию из металлического слоя и просветляющей прослойки. Выделив слой этой прослойки, прилегающий к просветляемому металлическому слою, обозначим его индексом I, остальную часть прослойки индексом 2 , а весь полуфильтр - ин-

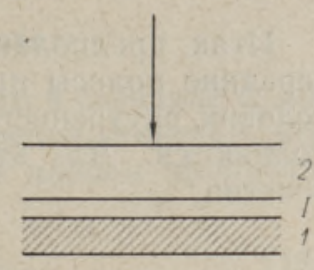
дексами 12. Схема фильтра изображена на фиг. 1.

Итак, перепишем формулы (1) и (4) в виде

$$
\begin{gathered}
\frac{1}{D}=\left(1+\frac{A_{12}}{D_{12}}\right)^{2}+\frac{4 R_{12}}{D_{12}^{2}} \sin ^{2}\left(\alpha-\mu_{12}+v_{12}\right), \\
\alpha-\mu_{12}+v_{12}=p \pi, \quad p \text {-целое. }
\end{gathered}
$$

Условия просветления металлического слоя 1 таковы (см. работу [1]):

$$
\cos \left(2 \alpha_{1}+\sigma_{1}-\mu_{2}+v_{2}\right)=-1
$$

H

$$
R_{2}=\frac{S_{1}-\operatorname{sh} \varphi_{1}}{S_{1}+\operatorname{sh} \varphi_{1}}
$$

где $\alpha_{1}, \mu_{2}$ и $v_{2}$ определены аналогично $\alpha, \mu_{1}$ и $v_{1}$ в формулах (2) и (3), а величины $S_{1}, \varphi_{1}$ и $\sigma_{1}$ определяются равенствами

$$
\begin{gathered}
S_{1}=\frac{1}{2}\left(b_{1} b_{1}^{*}-a_{1} a_{1}^{*}-\tilde{b}_{1} \tilde{b}_{1}^{*}+\tilde{a}_{1} \tilde{a}_{1}^{*}\right) \\
\operatorname{ch} \varphi_{1}=\frac{1}{2}\left(b_{1} \dot{b}_{1}^{*}-a_{1} a_{1}^{*}+\tilde{b}_{1} \tilde{b}_{1}^{*}-\tilde{a}_{1} \tilde{a}_{1}^{*}\right) \\
U_{1} e^{i \sigma_{1}}=\tilde{a}_{1} b_{1}-a_{1} \tilde{b}_{1},
\end{gathered}
$$

причем

$$
U_{1}=\sqrt{S_{1}^{2}-\operatorname{sh}^{2} \varphi_{1}} \text { * }
$$

* Заметим, что в этих формулах $a_{1}, b_{1}$ не совпадают с $a_{1}, b_{1}$ в формуле (3), так как там подложкой для металлического слоя была стеклянная пластинка, а теперь слой I. Старое обозначение чам более не понадобится, так что смешения опасаться не следует. 
Здесь звездочка * означает, что берутся комплексно-сопряженные величины, а - величины, относящиеся к сопряженному слою (см. paботы $\left.\left[{ }^{1,4}\right]\right)$. Если условия (8) и (9) выполнены, то

$$
\frac{A_{12}}{D_{12}}=\left(\frac{A_{12}}{D_{12}}\right)_{\min }=e^{\varphi_{1}}-1
$$

Итак, предполагая условия просветления (8) и (9) выполненными в середине полосы пропускания, т. е. при условии (7), покажем, что все условия внутреннего и внешнего просветления всего фильтра тоже выполняются. Для этого достаточно показать лишь справедливость равенства

$$
\cos \left(2 \alpha+2 \sigma_{1}^{\prime}\right)=1 \text {, }
$$

где штрих ' означает падение света в обратном направлении. Это равенство является одним из условий внутреннего просветления. Другое условие внутреннего просветления (см. работу [3]) выполняется уже в силу симметричности фильтра. Что же касается условий внешнего просветления, то, как показано в работе [3], они равносильны условиям просветления (8) и (9) одного слоя, если выполнены условия внутреннего просветления. Поэтому равенство (15) достаточно для полной просветленности фильтра.

Чтобы вывести равенство (15) из наших предпосылок (7)-(9), наf пишем известные формулы (см. работу [ $\left.{ }^{4}\right]$ )

$$
\begin{aligned}
& a_{12}=a_{1} b_{2} e^{i \alpha_{1}}+a_{2} \tilde{b}_{1}^{*} e^{-i \alpha_{1}}, \\
& b_{12}=b_{1} b_{2} e^{i \alpha_{1}}+a_{2} \tilde{a}_{1}^{*} e^{-i \alpha_{1}}
\end{aligned},
$$

откуда следует:

$$
\begin{aligned}
\frac{\sqrt{R_{12}}}{D_{12}} e^{i\left(\mu_{12}-v_{12}\right)}= & \left(a_{1} b_{2} e^{i \alpha_{I}}+a_{2} \tilde{b}_{1}^{*} e^{-i \alpha_{I}}\right)\left(b_{1}^{*} b_{2}^{*} e^{-i \alpha_{I}}+a_{2}^{*} \tilde{a}_{1} e^{i \alpha_{I}}\right)=\frac{a_{1} b_{1}^{*}}{D_{2}}+\frac{\tilde{a}_{1} \tilde{b}_{1}^{*} R_{2}}{D_{2}}+ \\
& +\frac{\sqrt{R_{2}}}{D_{2}}\left(a_{1} \tilde{a}_{1} e^{i\left(2 \alpha_{I}-\mu_{2}+v_{2}\right)}+b_{1}^{*} \tilde{b}_{1}^{*} e^{-i\left(2 \alpha_{1}-\mu_{2}+v_{2}\right)}\right) .
\end{aligned}
$$

Подставляя сюда $R_{2}=\frac{S_{1}-\operatorname{sh} \varphi_{1}}{S_{1}+\operatorname{sh} \varphi_{1}}$ и $D_{2}=\frac{2 \operatorname{sh} \varphi_{1}}{S_{1}+\operatorname{sh} \varphi_{1}}$ и учитывая формулу (13), а также (8), находим

$$
\begin{gathered}
\frac{\sqrt{R_{12}}}{D_{12}} e^{i\left(\mu_{12}-v_{12}\right)}=\frac{1}{2 \operatorname{sh} \varphi_{1}}\left[S_{1}\left(a_{1} b_{1}^{*}+\tilde{a}_{1} \tilde{b}_{1}^{*}\right)+\operatorname{sh} \varphi_{1}\left(a_{1} b_{1}^{*}-\tilde{a}_{1} \tilde{b}_{1}^{*}\right)-U_{1} e^{-i \sigma_{1}} a_{1} \tilde{a}_{1}-\right. \\
\left.-\quad-U_{1} e^{i \sigma_{1}} b_{1}^{*} \tilde{b}_{1}^{*}\right] .
\end{gathered}
$$

Вводя сюда вместо $S_{1}$ и $U_{1} e^{ \pm i_{1}}$ выражения этих величин, согласно формулам (10)-(12), раскрывая скобки и группируя члены вновь, находим

$$
\frac{\sqrt{R_{12}}}{D_{12}} e^{i\left(\mu_{12}-v_{12}\right)}=\frac{e^{\varphi_{1}}}{2 \operatorname{sh} \varphi_{1}}\left(a_{1} b_{1}^{*}-\tilde{a}_{1} \tilde{b}_{1}^{*}\right) .
$$

Но известно (см. работу $\left.\left[{ }^{4}\right]\right)$, что $a_{1}^{\prime}=-\tilde{a}_{1}^{*}$ и $b_{1}^{\prime}=b_{1}$, следовательно,

$$
\frac{\sqrt{R_{12}}}{D_{12}} e^{i\left(\mu_{12}-v_{12}\right)}=-\frac{U_{1}^{i} e^{-i \sigma_{1}^{*} e^{\varphi_{1}}}}{2 \operatorname{sh} \varphi_{1}} .
$$


Отсюда сразу вытекает

$$
e^{i\left(\mu_{12}-v_{12}+\sigma_{1}^{\prime}\right)}=-1
$$

С другой стороны, равенство (7) равносильно равенству

$$
e^{i\left(\alpha-\mu_{12}+v_{12}\right)}=(-1)^{p} .
$$

Следовательно, $e^{i\left(2 \alpha+2 \sigma_{1}^{\prime}\right)}=1$, что и требовалось доказать.

Итак, если условия (8) и (9) выполнены, то фильтр в середине полосы пропускания просветлен как внешне, так и внутренне. Конечно, эти условия должны быть выполнены также в середине полосы пропускания. Из формул (6) и (14) вытекает, что тогда

$$
D_{\max }^{\mathrm{npocB}}=e^{-2 \varphi_{1}} \text {. }
$$

Сравним этот результат с $D_{\max }$ непросветленного фильтра. Перепишем формулу (5) в виде

$$
D_{\max }=\frac{1}{\left(\operatorname{ch} \varphi_{1}+\mathrm{S}_{1}^{0}\right)^{2}},
$$

где $S_{1}^{0}$ означает то же, что $S_{1}$ в формуле $(10)$, но для непросветленного полуфильтра. Следовательно, коэффициент пропускания в максимуме увеличивается в результате просветления в

$$
\frac{D_{\max }^{\mathrm{npocB}}}{D_{\max }}=\left(\operatorname{ch} \varphi_{1}+S_{1}^{0}\right)^{2} e^{-2 \varphi_{1}}
$$

раз. В следующем разделе на примере мы увидим, что это число может быть внушительным.

Далее, формула (17) дает также сразу выражение для величины $4 R_{12} / D_{12}^{2}$, определяющей ширину полосы пропускания светофильтра

$$
\frac{4 R_{12}}{D_{12}^{2}}=\frac{U_{1}^{\prime 2} e^{2 \varphi_{1}}}{\operatorname{sh}^{2} \varphi_{1}} .
$$

Ниже увидим, что эта величина тоже может иметь достаточно большие значения.

Отметим, наконец, что коэффициент отражения просветленного фильтра в середине полосы пропускания равен нулю. В самом деле, из общей теории (см. работу $\left.\left[{ }^{3}\right]\right)$ известно, что

$$
\left(\frac{A}{D}\right)_{\min }=e^{2 \varphi_{1}}-1 \text {. }
$$

Отсюда и из формулы (18) следует, что $A+D=1$, т. е. $R=0$. Этот результат есть частный случай общего свойства просветленных поглощаюших симметричных пленок (см. работу [5]).

\section{3. Пример и обсуждение}

Примем показатель преломления металлических слоев равным $\eta_{1}-i \varkappa_{1}=0,27-2,5 i$. Это значение, как можно заключить из имеющихся в литературе данных (см. [6], стр. 276), близко к истинному для сло- 
ев серебра толщиной больше $150-200 \AA$. Величина $\varphi_{1}$, как известно (см. работу [2]), зависит только от самого поглощающего слоя, но не зависит от обрамляющих его сред. Поэтому $D_{\max }^{\operatorname{mpocs}}$ (см. формулу (18))

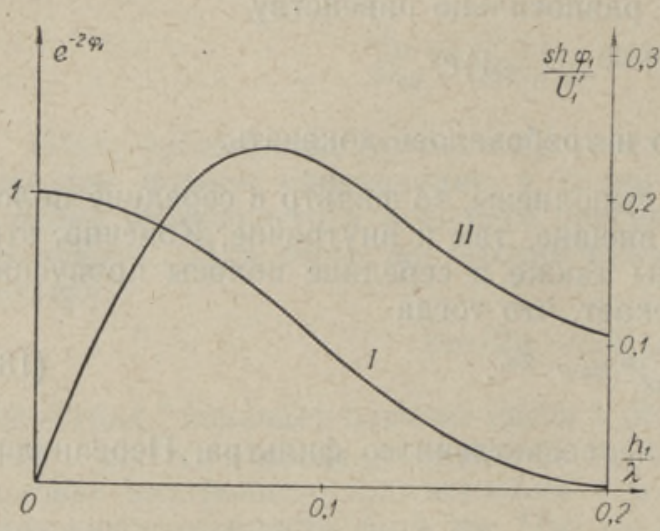

Фиг. 2. можно вычислить как функцию от толщины слоя серебра в долях длины волны (т. е. $\left.h_{1} / \lambda\right)$. На фиг. 2 дан график этой функции (кривая I). Задавая ту или иную толщину слоя, можем найти по графику сразу коэффициент пропускания фильтра в середине полосы пропускания.

Әффективность просветления зависит, согласно формуле $(20)$, от $\varphi_{1}$ и $S_{1}^{0}$. Последняя величина зависит не только от слоя серебра, но и от показателя преломления стеклянной пластинки. Примем его равным 1,65. Тогда по извест-

ным формулам (см. работу [ $\left.{ }^{1}\right]$ ) можно вычислить $S_{1}^{0}$ в зависимости от $h_{1} / \lambda$, и далее значения $D_{\max }^{\text {просв }} / D_{\max }$ для различных толщин слоя серебра. Результаты приведены в нижеследующей таблице:

$\begin{array}{llllll}h_{1} / \lambda & 0,04 & 0,06 & 0,08 & 0,10 & 0,12 \\ D_{\max }^{\text {npocs }} & 0,904 & 0,791 & 0,646 & 0,486 & 0,330 \\ D_{\max }^{\text {mpocs }} / D_{\max } & 1,409 & 1,710 & 2,229 & 3,188 & 4,927\end{array}$

Как видно из таблицы, просветление дает в самом деле значительный выигрыш в интенсивности.

Рассмотрим, наконец, вопрос о полуширине полосы пропускания. Эта величина определяется в зависимости от толщины слоев серебра функцией $\operatorname{sh} \varphi_{1} / U_{1}^{\prime}$, график которой (кривая II) приведен на фиг. 2. Точнее, эта функция определяет верхнюю границу для полуширины полосы пропускания. В самом деле, как вытекает из формул (6), (14) и (21), относительная полуширина. полосы пропускания выражается как

$$
\frac{\Delta \lambda}{\lambda}=\frac{\operatorname{sh} \varphi_{1}}{U_{1}^{\prime}\left(\pi p+\mu_{12}-v_{12}\right)}
$$

если не учитывать, во-первых, ухудшения условий просветления при изменении длины волны на $\Delta \lambda$ и, во-вторых, дисперсии величины $\mu_{12}-v_{12}$. Оба неучтенных фактора сужают фактически полосу пропускания, так что формула (22) дает, действительно, только верхнюю границу полуширины полосы пропускания.

Заметим, что $U_{1}^{\prime}$, в отличие от $\varphi_{1}$, зависит не только от слоя серебра, но и от показателя преломления промежуточного слоя, будучи тем больше, чем меньше $n$. Поэтому, чтобы $s h \varphi_{1} / U_{1}^{\prime}$ было возможно меньше, в нашем примере выбрано для $n$ возможно меньшее значение, именно $n=1,35$.

Из графика II на рис. 2 мы видим, что даже максимальное значение $\operatorname{sh} \varphi_{1} / U_{1}^{\prime}$, равное 0,23, сравнительно мало. Следовательно, уже при не слишком высоком порядке $p$ интерференции можно получить фильтр с довольно узкой полосой пропускания. Добавим еще, что путем введе- 
ния двух специально подобранных непоглощающих прослоек (одинако. вых, чтобы фильтр оставался симметричным) между промежуточным слоем и металлическими слоями можно увеличить $U_{1}^{\prime}$ без изменения $\varphi_{1}$. Однако на этой дополнительной возможности сужения полосы пропускания мы здесь останавливаться не будем, отсылая читателя к статье [7], в которой подробно рассматриваются сходные вопросы. Мы не будем также. касаться вопроса о возможном составе просветляющей прослойки (12 на фиг. 1), так как этот вопрос относится полностью к проблеме синтеза непоглощающего покрытия, имеющего заданные свойства (согласно формулам (8) и (9)), а эта проблема решается своими особыми методами, не имеюшими непосредственного отношения к проблеме просветления поглошающего фильтра.

\title{
Л И Т Е Р А Т У Р A
}

1. П Г. К а рд, Теория просветления металлическй покрытий, Оптика и спектроскопия,. т. 9 , № $2,1960$.

2. П. Г. К а рд, К теорни поглощающих оптических покрытий, Изв. АН ЭССР, серия физ -мат. и техн. наук, т. IX, № 3, 1960.

3. П. Г. К а рд, Основы теории синтеза просветленных поглощающих покрытий, Оптика и спектроскопия, т. 9, № 3, 1960.

4. П. Г. К а рд, Новые рекуррентные формулы в теории многослойных оптических покрытий, Оптика и спектроскопия, т. 9, № 1, 1960.

5. П. Г. К а рд, Некоторые свойства поглощающих оптических покрытий, Изв. АН ЭССР, серия физ.-мат, и техн. наук, т. ХІ, № 1, 1962.

6. Г. В. Р озен бе р г, Оптика тонкослойных покрытий, М., ГИФМЛ, 1958.

7. П. Г. К а рд, Теория многсслойных несимметричных отражателей, Оптика и спектроскопия, т. 10 , № $3,1961$.

Тартуский государственный университет

Постугила в редакцию

25. XII 1961

\section{FABRY-PEROT' TOOPI SELGENDATUD VALGUSFILTRI TEOORIA}

\author{
P. Kard, \\ Eesti NSV Teaduste Akadeemia korrespondeeriv liige \\ Resümee
}

Artiklis näidatakse, et sümmeetrilise Fabry-Perot' tüüpi valgusfiltri selgendamiseteel $\left[^{1-3}\right]$ on vôimalik oluliselt tōsta läbilaskvuse koefitsienti. Seejuures selgub, et filtri täielik (nii sisemine kui ka väline) selgendus läbilaskeriba keskkohas saavutatakse môlema poolfiltri selgendamise teel (vahekihist väljapoole suunatud valguse langemise juhuks). Selgendatud filtri läbilaskeriba laius osutub küllalt väikeseks ja teatud lisakihtide abil on teda võimalik teha veelgi kitsamaks läbilaskvuse koefitsienti mōjutamata. 


\section{A THEORY OF THE CLARIFIED LIGHT-FILTER OF THE FABRY-PEROT TYPE}

P. Kard,

Corresponding Member of the Academy of Sciences of the Estonian SSR

\section{Summary}

It is shown that the transmittance of the symmetrical light-filter of the Fabry-Perot type is possible to be considerably raised by means of clarification $\left[{ }^{1}-3\right]$. In addition it is proved that the thorough clarification (both the internal and the external) is obtained by the clarification of both half-filters (in the direction of incidence from the intermediate layer outside). The passband-width of the clarified filter turns out to be sufficiently small, and by means of certain additional layers it can be still reduced independently from the value of the transmittance.

Received

Dec. 25th, 1961 